\title{
First experimental data of sulphur ions sputtering water ice
}

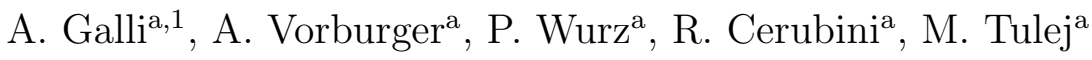 \\ ${ }^{a}$ Physikalisches Institut, University of Bern, Bern, Switzerland
}

\begin{abstract}
This paper presents the first experimental sputtering yields for sulphur ions with energies between $10 \mathrm{keV}$ and $140 \mathrm{keV}$ irradiating water ice films on a microbalance. The measured sputtering yields exceed theoretical predictions based on other ion species by a factor of 2 to 3 for most energies. Moreover, the sputtering yield of $\mathrm{SF}^{+}$molecules is compared to the yield of atomic species $\mathrm{S}^{+}$and $\mathrm{F}^{+}$. As found for atomic versus molecular oxygen, the sputtering yield caused by molecules is two times higher than expected. Finally, the implications of the enhanced sulphur sputtering yield for Europa's atmosphere are discussed.
\end{abstract}

Keywords: Ices, Jupiter satellites, Experimental techniques, Sputtering, Radiolysis

\section{Introduction}

When water ice is irradiated with energetic ions, the energy transferred from the impactor to the ice may eject particles from the surface. This process, termed sputtering, has been studied for several ion species and energy ranges under laboratory conditions over the past decades (e.g., Haring et al. (1984b) and accompanying papers, Baragiola et al. (2003), Farenzena et al. (2005), Famá et al. (2008), Johnson et al. (2009), Cassidy et al. (2013), Muntean et al. (2016), and Galli et al. (2017)). Such results are needed to check theoretical models of sputtering (Sigmund, 1969; Johnson, 1989, Cassidy and Johnson, 2005) and relate them to observations (Hall et al., 1995; Hansen et al., 2005; Roth et al., 2016) and models (Shematovich et al., 2005 ,

\footnotetext{
${ }^{1}$ Corresponding author, E-mail address: andre.galli@space.unibe.ch
} 
Smyth and Marconi, 2006; Plainaki et al., 2012; Vorburger and Wurz, 2018) of sputter-induced atmospheres of icy moons such as Europa.

Sputtering may proceed in a straight-forward manner, i.e., the ion directly knocking out one or several water molecules, or it may be a two-stage process with the irradiation first causing chemical reactions inside the ice (socalled radiolysis (Haring et al., 1984a; Johnson et al., 2004, Cassidy et al., 2010) ) and subsequently releasing the radiolysis products from the surface. The sputtering yield denotes in both cases the number of water molecules or equivalents (if $\mathrm{H}_{2} \mathrm{O}$ reacted to $\mathrm{H}_{2}$ and $\mathrm{O}_{2}$, for instance) leaving the ice per impacting particles. Knowing this yield and the chemical and energetic composition of the ejecta over a wide range of parameters is important to understand any ice-covered celestial body. The astrophysical application we are most interested here is Europa, one of the icy moons of Jupiter. For these bodies, the sputtering yields and the plasma environment determine the density and composition of their atmospheres (see Johnson et al. (2004) for a review).

For this study, we sputtered thin water ice films with sulphur ions and sulphur-bearing molecules from a microbalance. This is the most common experimental method used so far (see for example Baragiola et al. (2003); Teolis et al. (2005); Famá et al. (2008); Muntean et al. (2016); Galli et al. (2017)). However, to our knowledge, sulphur ions were never used before in such experiments as sulphur is chemically reactive and can corrode surfaces in vacuum chambers. Argon was the species closest in mass to sulphur so far, for which experimental results exist (see compilations by Johnson et al. (2009); Cassidy et al. (2013)). Knowing the sputtering yield of $\mathrm{S}^{+}$on water ice is highly relevant for Europa's atmosphere: $\mathrm{S}^{+}$ions are one of the three most frequent ion species in the plasma environment around Europa (Paranicas et al., 2002) because of its volcanically active neighbour Io.

After a recapitulation of the theory of ice sputtering (Section 2), we describe the experiment set-up in Section 3. We then present the sputtering results for $\mathrm{S}^{+}, \mathrm{F}^{+}$, and $\mathrm{SF}^{+}$molecules (Section 4). The paper is completed with a discussion on the implications of these results for Europa and for future laboratory work (Section 5), followed by the conclusions (Section 6).

\section{Theory}

We will compare our new experimental results for $\mathrm{S}^{+}$and $\mathrm{F}^{+}$to two widely used semi-empirical formulae for ion sputtering yield derived by Famá et al. 
(2008) and Johnson et al. (2009). These formulae are based on previous experiments with other ion species irradiating dense water ice films. For ion energies below $10 \mathrm{keV}$, the sputtering yield can be described by a cascade of elastic collisions, whereas the so-called electronic sputtering dominates at higher energies. Equation 1 by Famá et al. (2008) is more accurate for lower energies; for energies above $100 \mathrm{keV}$, Equation 2 by Johnson et al. (2009) offers a better fit to experimental data for $\mathrm{H}^{+}, \mathrm{He}^{+}, \mathrm{N}^{+}, \mathrm{O}^{+}, \mathrm{Ne}^{+}$, and $\mathrm{Ar}^{+}$ ion beams (Cassidy et al. 2013):

$Y\left(E, m_{1}, Z_{1}, \theta, T\right)=\frac{1}{U_{i}}\left(\frac{3}{4 \pi^{2} C_{0}} \alpha S_{n}+\eta S_{e}^{2}\right)\left(1+q_{i} \exp \left(-\frac{E_{a}}{k_{B} T}\right)\right) \cos ^{-f}(\theta)$

Equation 1 quantifies the sputtering yield as a sum of nuclear and electronic sputtering, described by the nuclear stopping cross section $S_{n}\left(E, m_{1}, Z_{1}\right)$ $=d E_{n} /(N d x)$ and the electronic stopping cross section $S_{e}\left(E, m_{1}, Z_{1}\right)$. The yield in Eq. 1 depends on energy $E$, mass of impactor $m_{1}$, atomic number of impactor $Z_{1}$, the incidence angle $\theta$ relative to the surface normal, and surface temperature $T$. The temperature-dependent term with the activation energy $E_{a}$ (Reimann et al., 1984) becomes dominant above $T=120 \mathrm{~K}$ and is due to radiolysis and subsequent release of $\mathrm{H}_{2}$ and $\mathrm{O}_{2}$ (Johnson et al., 2004; Famá et al., 2008; Teolis et al., 2009). This contribution makes up only $10 \%$ of $Y$ at $90 \mathrm{~K}$ (Equation 1); it rises to $20 \%$ at $100 \mathrm{~K}$ once the ice has been saturated with $\sim 10^{15}$ ions $\mathrm{cm}^{-2}$ (Teolis et al., 2005). For $U_{i}$, the sublimation energy of water $(0.45 \mathrm{eV})$ is assumed. The effective cross-section for low energy recoils, $C_{0}=1.3 \AA^{2}$, the activation energy, $E_{a}=0.06 \pm 0.01 \mathrm{eV}$, and $q_{i}=220$ are constants (Famá et al., 2008). The parameter describing the angular dependence calculates to $f=1.75$ for $\mathrm{S}^{+}$.

By comparison, Johnson et al. (2009) propose the following empirical formula for the sputtering yield (the angular and temperature dependence are identical to Equation [1):

$$
Y\left(E, m_{1}, Z_{1}, \theta, T\right)=1 /\left(1 / Y_{\text {low }}+1 / Y_{\text {high }}\right)\left(1+q_{i} \exp \left(-\frac{E_{a}}{k_{B} T}\right)\right) \cos ^{-f}(\theta)
$$

whereby $Y_{\text {low }}$ and $Y_{\text {low }}$ stand for:

$$
Y_{i}=Z_{1}^{2.8} C_{1}\left(\frac{v}{2.19 \times 10^{6}} Z_{1}^{-1 / 3}\right)^{C_{2}} \text {, with ion velocity } v=\sqrt{2 E / m_{1}}
$$

The fit parameters are $C_{1}=4.2$ and $C_{2}=2.16$ for $Y_{\text {low }}$ and $C_{1}=11.22$ and $C_{2}=-2.24$ for $Y_{h i g h}$. 


\section{Experiment set-up}

The same microbalance set-up was used as in our previous ice film experiments (Galli et al., 2017): We background deposited de-ionized water vapour via a needle valve and a capillary onto the cooled surface of a microbalance in a vacuum chamber. The sensitivity of the microbalance was $1.61 \times 10^{9} \mathrm{~Hz} \mathrm{~g}^{-1}$ according to calibration in 2014 performed by the manufacturer (gold-coated $15 \mathrm{MHz}$ quartz crystal, manufacturer: QCM Research). The surface of the microbalance was $45^{\circ}$ or $60^{\circ}$ tilted with respect to the incoming ion beam. Under these conditions and temperatures around $90 \mathrm{~K}$, most of the deposited ice will remain amorphous throughout the experiments and the porosity will vary between few \% (Famá et al., 2008) and 25\% (Mitchell et al., 2017). If the bulk density is $0.9 \mathrm{~g} \mathrm{~cm}^{-3}$, one monolayer of $\mathrm{H}_{2} \mathrm{O}$ on the microbalance corresponds to a frequency shift of $14 \mathrm{~Hz}$. The $\mathrm{H}_{2} \mathrm{O}$ partial pressure in the chamber ranged from $3 \times 10^{-8}$ to $4 \times 10^{-7}$ mbar during vapour deposition; the ice film accretion rate increased linearly with that partial pressure from 0.2 to $5 \mathrm{~Hz} \mathrm{~s}^{-1}$. Within this range, the deposition rate did not notably affect the measured sputtering yields. For irradiation experiments, the ice film thickness ranged from 40 to $200 \mathrm{~nm}$ and the residual water pressure in the vacuum chamber was only on the order of $10^{-9}$ mbar.

To create an ion beam, we ionized $\mathrm{SF}_{6}$ gas and accelerated the ion species with an electron-cyclotron-resonance ion source (Marti et al., 2001). The ion source produced many different species from the parent molecule $\mathrm{SF}_{6}$, but only $\mathrm{S}^{+}, \mathrm{F}^{+}, \mathrm{S}^{2+}$ and $\mathrm{SF}^{+}$turned out to have a sufficiently high beam current to create a detectable sputtering signal when the ion beam was directed at the water ice film on the microbalance. The beam currents reached 0.1 to 1.0 $\mathrm{nA}$, which corresponded, at a beam diameter of $0.3 \mathrm{~cm}$, to $(0.9 \ldots 9) \times 10^{10}$ ions $\mathrm{cm}^{-2} \mathrm{~s}^{-1}$. To interpret the results for the $\mathrm{SF}_{6}$ fragments and to verify the microbalance sensitivity, we also irradiated the microbalance with $\mathrm{O}^{+}$ ions whose sputtering yield is well known from previous studies (Shi et al., 1995; Baragiola et al., 2003).

\section{Results}

\subsection{Accuracy of results}

Before we discuss the results for the hitherto unknown sputtering yields of $\mathrm{S}^{+}, \mathrm{F}^{+}$, and $\mathrm{SF}^{+}$, let us first assess the general accuracy of our experiments using oxygen as a reference. Oxygen sputtering yields have been measured 
numerous times by us and other research groups whereby Equation 1 fits most previous laboratory experiments within 30\% relative uncertainty (Famá et al., 2008). We therefore collected all $\mathrm{O}^{+}$yield results measured at an impact angle of $45^{\circ}$ and 10,30 , and $50 \mathrm{keV}$ energy over the last 1.5 years in our facility. This data set was accumulated during five different measurement series separated by several weeks or months. The first part of this data set covering the year 2016 was presented in Galli et al. (2017); here we added the measurements from 2017. We normalized all data (obtained at temperatures between 89 and $101 \mathrm{~K}$ ) to the same temperature $T=90 \mathrm{~K}$ assuming the temperature dependence in Equations 1 and 2. Apart from temperatures also vacuum pressure, vapour deposition rate, ice film thickness, and irradiation duration varied. Moreover, we used two different microbalances of the same type (see Section 3).

The average sputtering yields derived from this comprehensive data set compared to the $Y_{\text {th }}$ predicted from Equation 1 (with $T=90 \mathrm{~K}$ ) as follows: $Y=44 \pm 13$ (14 data points) vs. $Y_{t h}=27$ at $10 \mathrm{keV}, Y=73_{-25}^{+14}$ (7 data points) vs. $Y_{t h}=62$ at $30 \mathrm{keV}$, and $Y=111_{-33}^{+15}$ (5 data points) vs. $Y_{t h}=105$ at $50 \mathrm{keV}$. The experimental error bars denote the ranges between average and most extreme positive and negative outlier.

These results are important in two respects: First, the experimental values are reproducible within $30 \%$ or better on the long run. During one measurement series, the scatter usually was on the order of 10\% (Galli et al., 2017). Second, the oxygen results agree with Equation 1, which reproduces previous results from other groups within 30\% (Famá et al., 2008). We will therefore attribute an uncertainty of $30 \%$ to our experimental yields for other ion species in the following section.

\subsection{Results for $\mathrm{S}^{+}, \mathrm{F}^{+}$, and $\mathrm{SF}^{+}$}

105 individual irradiations with $\mathrm{S}^{+}, \mathrm{SF}^{+}, \mathrm{S}^{2+}$, and $\mathrm{F}^{+}$ions hitting the ice film were performed during two different measurement series of six days in total. 11 of the $\mathrm{S}^{+}$irradiations took place the same day when also $\mathrm{O}^{+}$ sputtering yields were measured for cross-calibration (see Section 4.1). The median temperature of the water ice film during the measurements was $90 \mathrm{~K}$ with extremes of $89 \mathrm{~K}$ and $101 \mathrm{~K}$. For evaluation, individual sputtering yields obtained at a temperature other than $90 \mathrm{~K}$ were normalized to $T=90 \mathrm{~K}$ based on Equations 1 and 2, At the given temperature range this corresponds to a modification by a factor of 1.1 at most. An individual irradiation lasted between 1 and 30 minutes; Fig. 1 shows as an example the frequency response 
of the microbalance (in $\mathrm{Hz}$ versus minutes) to a $50 \mathrm{keV} \mathrm{S}^{+}$ion beam for 2 and 1 minutes of irradiation. With these measurements, we derived the sputtering yield from the difference of the accretion rate during irradiation compared to the rate before and after irradiation when the ion beam was off.

After depositing an ice film, we irradiated it several times to reach fluences of $1.8 \times 10^{14}$ ions $\mathrm{cm}^{-2}$ at most. We then either deposited a fresh ice film onto the irradiated ice film (repeating this step one or two times) or we desorbed all ice layers before depositing a pristine ice film by heating and cooling the microbalance. The derived sputtering yields did not change notably for the two different cases. Due to the build-up of $\mathrm{O}_{2}$ in the ice film, irradiation at higher ion fluence results in higher sputtering yields (Teolis et al., 2005). At $90 \mathrm{~K}$ ice temperature, however, this contribution is expected to enhance the total sputtering yield by only $10 \%$ for saturated ice. Moreover, the highest ion fluence in our measurements corresponds to only $20 \%$ of the saturation fluence (see Section 2). We quantified the fluence effect on sputtering yields in two ways: During 30 minutes of continuous irradiation with a $30 \mathrm{keV} \mathrm{S}+$ beam, the sputtering yields varied by less than $10 \%$. We also compared the sputtering yields from subsequent irradiations at identical parameters. For 43 different pairs of irradiations, the second attempt resulted in a $1.06 \pm 0.09$ higher sputtering yield than the first attempt. In summary, varying ion fluences affected the sputtering yields presented here by $10 \%$ at most. This agrees with the estimate for $\mathrm{O}^{+}$and $\mathrm{Ar}^{+}$irradiation (Galli et al., 2017). Hence we did not discriminate data according to fluence and organized the data points solely according to different ion species, impact angle $\left(45^{\circ}\right.$ and $30^{\circ}$ relative to surface normal), and energy (10 keV to $140 \mathrm{keV})$. This approach resulted in 22 different settings to be distinguished.

The effect of ice film thickness is taken into account the same way as in Galli et al. (2017): We relied on SRIM calculations (Ziegler et al., 1985, 2008) to estimate the average penetration depth of ions. In the results (Table 1), we included only those sputtering yields measured with an ice film thickness deeper than the penetration depth: sputtering yields derived from irradiations at an insufficient film thickness result in a notably larger scatter (refer to Fig. 1 in Galli et al. (2017)). The only exception was the measurement with $140 \mathrm{keV} \mathrm{S}^{2+}$. Here, the ice film was only 0.4 times the expected penetration depth. The study by Galli et al. (2017) about $\mathrm{Ar}^{+}$and $\mathrm{O}^{+}$sputtering showed that the measured sputtering yield for irradiations with that ratio of ice film thickness to penetration depth was 0.9 times the value measured for thicker ice films. The yield and its uncertainty for $140 \mathrm{keV} \mathrm{S}^{2+}$ therefore 


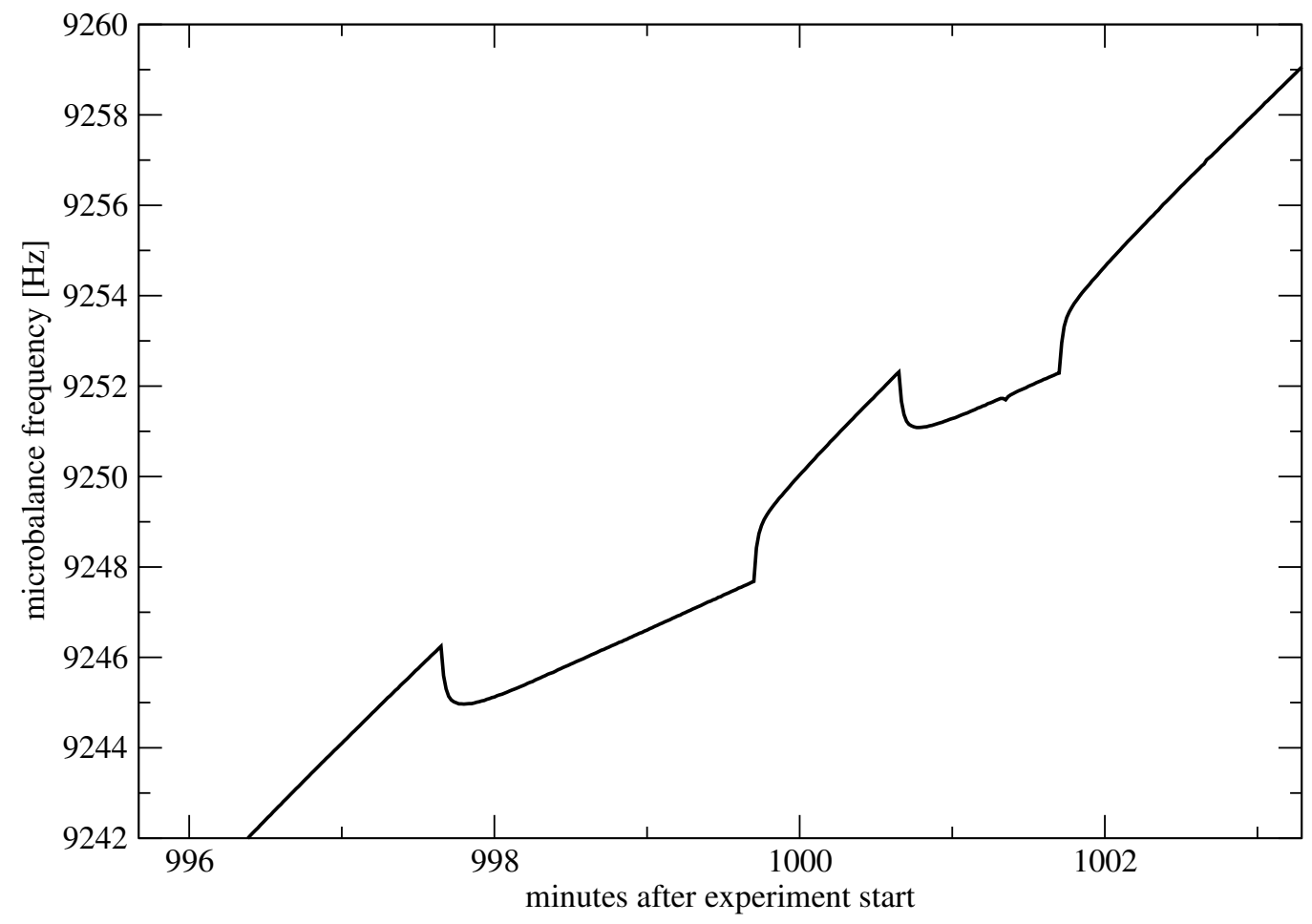

Figure 1: Frequency response of the microbalance to a $50 \mathrm{keV} \mathrm{S}^{+}$ion beam for 2 and $1 \mathrm{~min}$ of irradiation. The ice accretion rate on the microbalance flattens because of the sputtering losses. The momentum of the ion beam also causes an immediate frequency drop (at minute 998 and 1001) when the ion beam hits the microbalance and a frequency rise when the ion beam is shut off (at minute 1000 and 1002). 
might be larger than stated in Table 1. For the $\mathrm{SF}^{+}$molecule, we assumed that it fragments upon impact and then used the kinetic energy of the $\mathrm{S}$ fragment (44 keV for the case of a $70 \mathrm{keV} \mathrm{SF}^{+}$molecule, e.g.) to estimate the penetration depth.

Regarding $\mathrm{F}^{+}$, only for one setting with $30 \mathrm{keV}$ did we achieve a thickness $\approx$ penetration depth. The values in Table 1 are the averages over the individual irradiations, the error bar is always $30 \%$ as derived in Section 4.1.

The resulting 22 values of $Y$ for the different data sets are presented in Table 1. The impact angle $\theta$ is the angle relative to the surface normal as in Equation 1. The energy in Table 1 is the total kinetic energy per ion, corrected for ion charge state. Based on previous experiments (Muntean et al. 2016, Galli et al. 2017), we assumed that charge state has no effect on sputtering yield and used Eq. 1 to predict the sputtering yield for $140 \mathrm{keV}$ $\mathrm{S}^{2+}$ accordingly. The nuclear and electronic stopping cross-sections, $S_{n}$ and $S_{e}$, were added for $\mathrm{S}^{+}$and $\mathrm{F}^{+}$in units of $\mathrm{eV} \AA^{2}$ following the approach in the Appendix of Famá et al. (2008).

The sputtering yields of $\mathrm{S}^{+}$and $\mathrm{SF}^{+}$increase with roughly $\cos ^{-1}(\theta)$ (see Equations 1 and 2) for an increase of impact angle $\theta$ from $30^{\circ}$ to $45^{\circ}$. This is a weaker dependence than the range of $f=1.3 \ldots 1.8$ found by Famá et al. (2008) and Vidal et al. (2005) for a wider range of angles and impactors. Since we have only two different angular positions at our disposal, we cannot conclude if the angular dependence for $\mathrm{S}^{+}$and $\mathrm{SF}^{+}$truly deviates from the hitherto established angular dependence for other sputtering species.

Figure 2 compares the experimental $\mathrm{S}^{+}$sputtering yields to the predictions of Eq. 1 (Famá et al. 2008) (blue curve) and Eq. 2 (Johnson et al., 2009) (red dashed curve) for the impact angle of $45^{\circ}$. The experimental sputtering yield exceeds the predictions by Famá et al. (2008) by a factor of $2.9 \pm 0.5$ for all energies between 20 and $70 \mathrm{keV}$. The discrepancy is less notable for 10 and $140 \mathrm{keV}$. By comparison, the formula by Johnson et al. (2009) matches the data at 30 and $50 \mathrm{keV}$. For lower energies where elastic collisions dominate, the predictions by Johnson et al. (2009) also underestimate the sputtering yield by a factor of two. The tendency of Equation 2 to underestimate yields at low energies was already noted by the authors and by Cassidy et al. (2013). More noteworthy is that the sputtering yield between 10 and $70 \mathrm{keV}$ exceeds predictions from Equation 1. That equation matches experiments better than Equation 2 below $100 \mathrm{keV}$ energy in the case of $\mathrm{H}^{+}, \mathrm{He}^{+}, \mathrm{N}^{+}, \mathrm{O}^{+}$, and $\mathrm{Ar}^{+}$(Cassidy et al. 2013$)$. The implications of these enhanced sulphur sputtering yields for Europa's atmosphere will be 
Table 1: Average sputtering yields $Y$ for all different experiment settings: ion species, energy $E$, and impact angle $\theta$. The theoretical values $Y_{t h}$ were calculated with the semiempirical formula in Eq. 1, assuming charge state does not affect sputtering yields. Data and theoretical values were scaled to the same temperature $T=90 \mathrm{~K}$ with Equations 1 and 2. The yield for $\mathrm{S}^{2+}$ with the asterisk might be $10 \%$ larger because of the insufficient ice film thickness at these high energies. No theoretical predictions for molecules such as $\mathrm{SF}$ exists yet. The average penetration depth and its uncertainty are listed as $d$ and $\sigma_{d}$, followed by the nuclear and electronic stopping cross-sections $S_{n}$ and $S_{e}$ from Eq. 1 .

\begin{tabular}{lrrrrrrrrr}
\hline Ion & $E(\mathrm{keV})$ & $\theta\left(^{\circ}\right)$ & $Y$ & $\sigma_{Y}$ & $Y_{t h}$ & $d(\mathrm{~nm})$ & $\sigma_{d}(\mathrm{~nm})$ & $S_{n}\left(\mathrm{eV} \AA^{2}\right)$ & $S_{e}\left(\mathrm{eV} \AA^{2}\right)$ \\
$\mathrm{S}^{+}$ & 70 & 45 & 144 & 43 & 52 & 104 & 39 & 27 & 17 \\
$\mathrm{~S}^{+}$ & 50 & 45 & 105 & 32 & 43 & 79 & 28 & 30 & 14 \\
$\mathrm{~S}^{+}$ & 30 & 45 & 94 & 28 & 35 & 56 & 20 & 33 & 10 \\
$\mathrm{~S}^{+}$ & 20 & 45 & 78 & 23 & 32 & 35 & 14 & 35 & 8 \\
$\mathrm{~S}^{+}$ & 10 & 45 & 46 & 14 & 28 & 22 & 9 & 35 & 5 \\
$\mathrm{~S}^{+}$ & 70 & 30 & 110 & 33 & 36 & 129 & 41 & 27 & 17 \\
$\mathrm{~S}^{+}$ & 50 & 30 & 98 & 29 & 30 & 96 & 30 & 30 & 14 \\
$\mathrm{~S}^{+}$ & 30 & 30 & 80 & 24 & 24 & 60 & 20 & 33 & 10 \\
$\mathrm{~S}^{+}$ & 20 & 30 & 65 & 20 & 22 & 42 & 15 & 35 & 8 \\
$\mathrm{~S}^{+}$ & 10 & 30 & 43 & 13 & 20 & 24 & 9 & 35 & 5 \\
$\mathrm{~S}^{2+}$ & 140 & 45 & $* 170$ & 51 & 94 & 213 & 67 & 21 & 26 \\
$\mathrm{SF}^{+}$ & 70 & 45 & 287 & 86 & $\mathrm{~N} / \mathrm{A}$ & 70 & 27 & $\mathrm{~N} / \mathrm{A}$ & $\mathrm{N} / \mathrm{A}$ \\
$\mathrm{SF}^{+}$ & 50 & 45 & 265 & 80 & $\mathrm{~N} / \mathrm{A}$ & 58 & 20 & $\mathrm{~N} / \mathrm{A}$ & N/A \\
$\mathrm{SF}^{+}$ & 30 & 45 & 234 & 70 & $\mathrm{~N} / \mathrm{A}$ & 34 & 14 & $\mathrm{~N} / \mathrm{A}$ & N/A \\
$\mathrm{SF}^{+}$ & 20 & 45 & 157 & 47 & $\mathrm{~N} / \mathrm{A}$ & 24 & 10 & $\mathrm{~N} / \mathrm{A}$ & $\mathrm{N} / \mathrm{A}$ \\
$\mathrm{SF}^{+}$ & 10 & 45 & 112 & 34 & N/A & 14 & 6 & N/A & N/A \\
$\mathrm{SF}^{+}$ & 70 & 30 & 229 & 69 & N/A & 87 & 28 & N/A & N/A \\
$\mathrm{SF}^{+}$ & 50 & 30 & 206 & 62 & N/A & 70 & 21 & N/A & N/A \\
$\mathrm{SF}^{+}$ & 30 & 30 & 186 & 56 & N/A & 36 & 14 & N/A & N/A \\
$\mathrm{SF}^{+}$ & 20 & 30 & 142 & 43 & N/A & 29 & 11 & N/A & N/A \\
$\mathrm{SF}^{+}$ & 10 & 30 & 102 & 31 & N/A & 17 & 7 & N/A & N/A \\
$\mathrm{F}^{+}$ & 30 & 45 & 66 & 20 & 53 & 84 & 34 & 14 & 9 \\
& & & & & & & & &
\end{tabular}




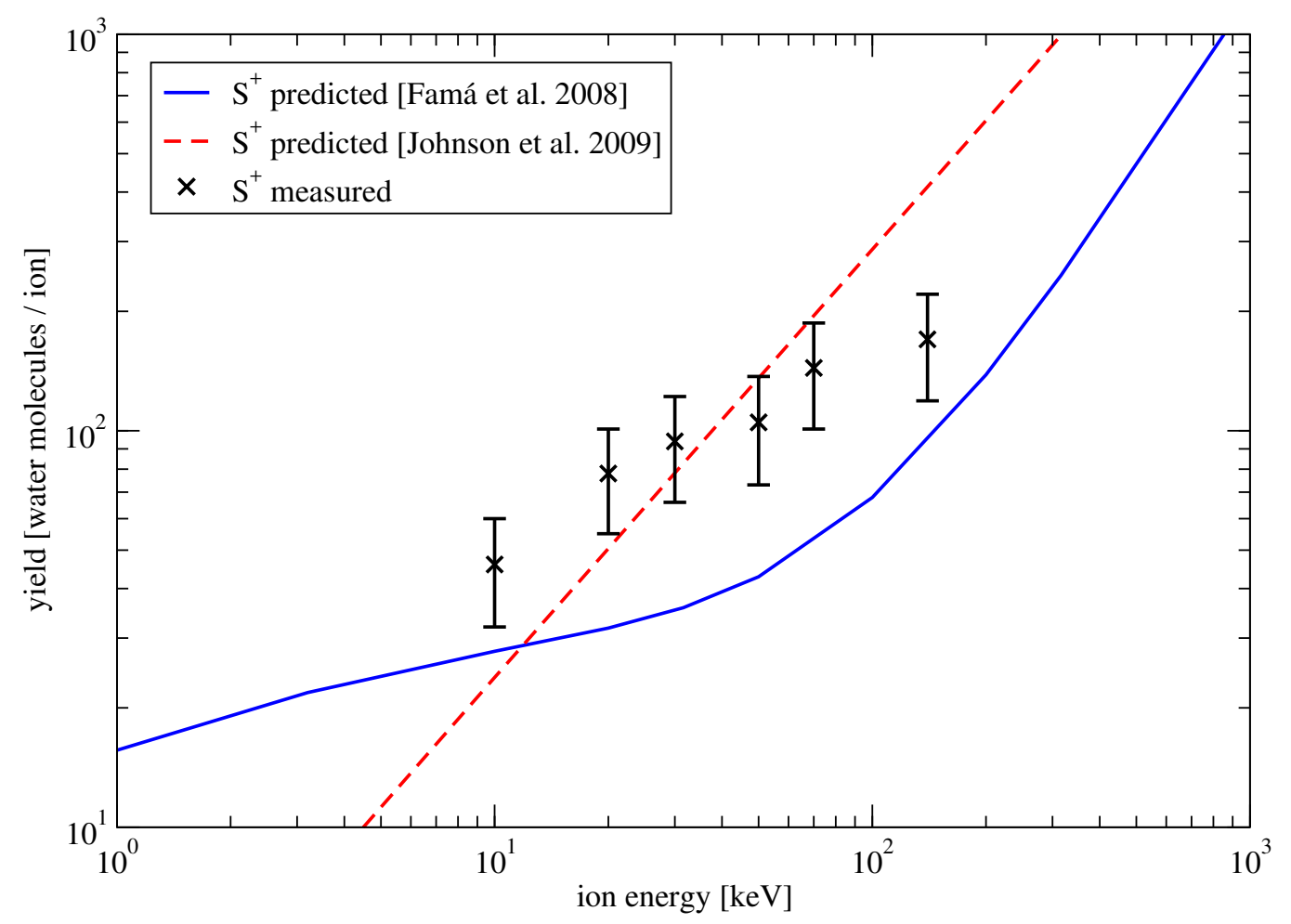

Figure 2: $\mathrm{S}^{+}$sputtering yields versus energy for $45^{\circ}$ impact angle and $T=90 \mathrm{~K}$. Data points (black symbols) are taken from the present study; the blue curve shows the prediction by Famá et al. (2008) and the red dashed curve shows the prediction by Johnson et al. (2009), both of which are based on previous ice sputtering experiments with other ion species.

discussed in Section 5 ,

The one experiment result for $\mathrm{F}^{+}$at $30 \mathrm{keV}$ agrees with predictions by Famá et al. (2008).

The measured sputtering yields for $\mathrm{SF}^{+}$are much higher than those for $\mathrm{S}^{+}$and $\mathrm{F}^{+}$at the same energies and impact angles. A quantitative interpretation of the $\mathrm{SF}^{+}$yields is difficult because they have never been measured before. Moreover, there is, to our knowledge, no general theory predicting sputtering yields from molecules irradiating solid surfaces. Comparing sputtering yields from atomic and molecular oxygen in water ice (Galli et al., 2017), we noted that the measured sputtering yield for an oxygen molecule was roughly $4 Y(E / 2)$ for energies between 10 and $50 \mathrm{keV}$. This is two times 
higher than the $2 Y(E / 2)$ one would predict under the assumption that the impact fragments the $\mathrm{O}_{2}$ molecule and distributes the available kinetic energy equally unto the fragments.

In the present study, we find a similar excess for $Y\left(\mathrm{SF}^{+}\right)$compared to the sputtering with atomic $\mathrm{S}^{+}$and $\mathrm{F}^{+}$. We considered the $\mathrm{SF}^{+}$measurements at an impact angle of $45^{\circ}$ in Table 1 and assumed that the kinetic energy of the molecule is re-distributed to its fragments upon impact according to the mass ratio. This implies, e.g., that a $30 \mathrm{keV} \mathrm{SF}^{+}$is fragmented into an $11 \mathrm{keV} \mathrm{F}^{+}$ and a $19 \mathrm{keV} \mathrm{S}^{+}$. We then applied Equation 1, multiplied by the observed enhancement of 1.25 , to calculate $Y\left(\mathrm{~F}^{+}\right)$and we interpolated $Y\left(\mathrm{~S}^{+}\right)$from Figure 2 to the relevant energies. The sum of the thus calculated monoatomic yields $Y\left(\mathrm{~S}^{+}\right)+Y\left(\mathrm{~F}^{+}\right)$is $83,110,141$, and 159 for molecule energies of 20,30 , 50 , and $70 \mathrm{keV}$, respectively. The ratio of measured molecular yields $\left(Y\left(\mathrm{SF}^{+}\right)\right.$ in Table 1) to these predictions calculates to $1.93 \pm 0.14$. The physical reason for this enhancement is currently unknown.

\section{Implications for Europa}

If sputtering yields of energetic sulphur ions irradiating water ice are higher than assumed so far, the density of molecules in Europa's atmosphere predicted by models will increase. We estimate this increase in this section. The main plasma constituents irradiating Europa's icy surface are electrons, $\mathrm{H}^{+}, \mathrm{O}^{+}$, and (from the nearby moon Io) $\mathrm{S}^{+}$(Ip et al., 1998; Cooper et al. 2001; Paranicas et al., 2002). All of these species form two separate distributions, a cold population with modes typically around $1 \mathrm{keV}$, and a hot population with modes around $100 \mathrm{keV}$. This is illustrated in Fig. 3 from Vorburger and Wurz (2018). Ion intensity measurements (red, blue, and green diamonds) were taken from Ip et al. (1998) whereas electron intensity measurements (black diamonds) were taken from Paranicas et al. (2001). The cold plasma energy spectra were fitted with drifting Maxwellian distributions (dashed lines) whereas the hot plasma energy spectra were fitted with Kappa distributions (solid lines). Also shown, for comparison, is the electron spectral shape as presented in Paranicas et al. (2001) (dotted black lines).

According to the model by Vorburger and Wurz (2018), cold ions, hot ions, cold electrons, and hot electrons all sputter the same order of magnitude $\mathrm{H}_{2}$ and $\mathrm{O}_{2}$ into the atmosphere, leading to a surface particle density between $10^{3}$ and $10^{4} \mathrm{~cm}^{-3}$ for $\mathrm{O}_{2}$ and $\mathrm{H}_{2}$. In addition, the cold and hot ions also eject $\mathrm{H}_{2} \mathrm{O}$ with a similar surface density. As a simple sensitivity test, 


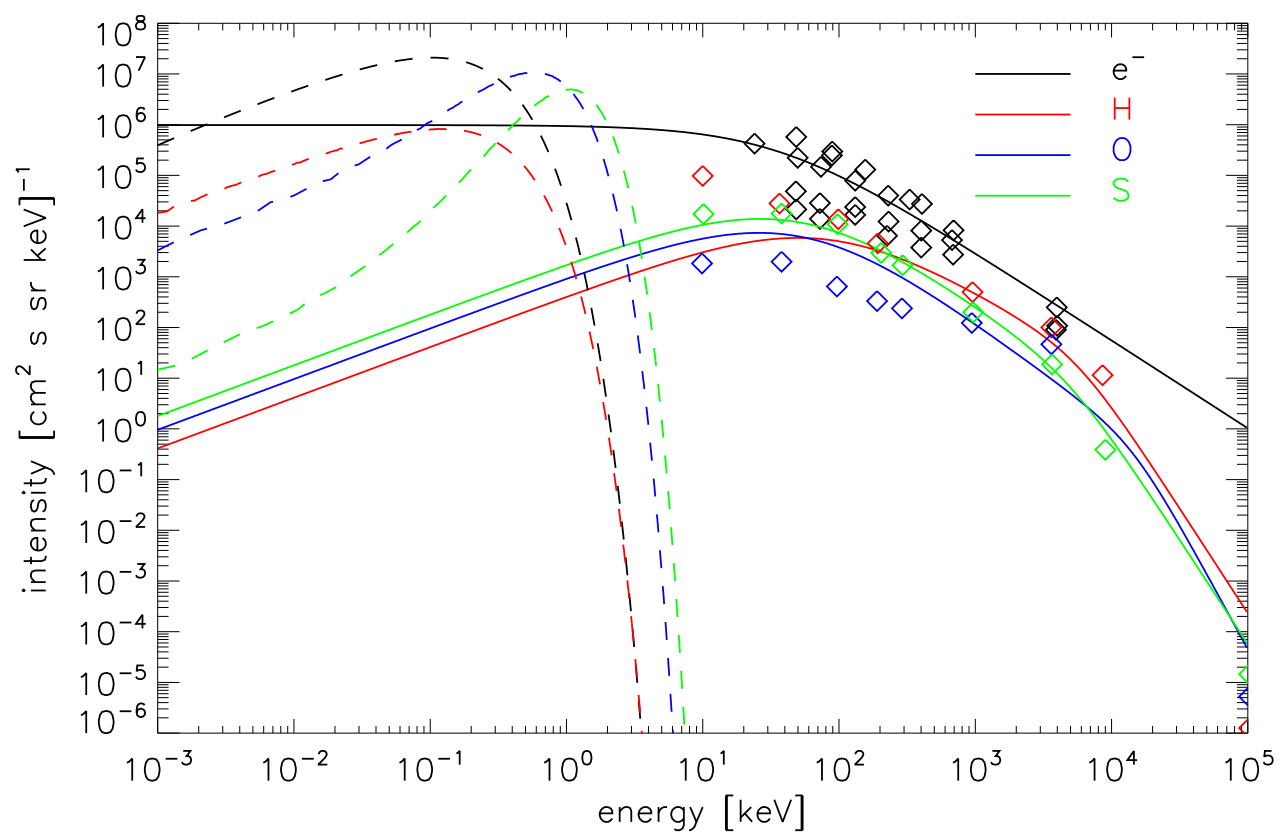

Figure 3: Intensity spectra of the four major plasma species (black: electrons, red: $\mathrm{H}^{+}$, blue: $\mathrm{O}^{+}$, green: $\left.\mathrm{S}^{+}\right)$at Europa's surface. The diamonds denote data from Ip et al. (1998) (ions) and Paranicas et al. (2001) (electrons), the solid lines are fits to the hot populations, the dashed lines are models of the cold populations that have not been measured yet. Figure adapted from Vorburger and Wurz (2018). 
we increased the sputtering yields for the hot sulphur population (between a few $\mathrm{keV}$ and a few $100 \mathrm{keV}$ ) by a factor of 3 compared to assumptions so far. This enhancement is consistent with the new experimental results. The sputtering yields for the cold sulphur population and for all other plasma species remained the same in the model. This would have the following impacts on the model predictions by Vorburger and Wurz (2018): The total surface density (average temperature assumed to be $106 \mathrm{~K}$ ) of the sputtered species at Europa increase from 1.0 to $1.4 \times 10^{4} \mathrm{~cm}^{-3}\left(\mathrm{H}_{2} \mathrm{O}\right), 0.9$ to $1.1 \times 10^{4} \mathrm{~cm}^{-3}$ $\left(\mathrm{H}_{2}\right)$, and from 1.6 to $1.9 \times 10^{4} \mathrm{~cm}^{-3}\left(\mathrm{O}_{2}\right)$. The atmosphere surface densities increase only by $30 \%$ in total because sulphur is not the only sputtering agent at Europa's surface.

This discussion only considered the effect of sulphur sputtering for the ejected particles and thus for Europa's atmosphere. The effects of sulphur ion irradiation on the chemical and physical properties of Europa's surface will be studied in future tests with chemically more complex ice films and with thick ice samples (Galli et al., 2016).

\section{Conclusions}

The first experimental results for sulphur ions sputtering of water ice show that the sputtering yield between 20 and $70 \mathrm{keV}$ is two to three times higher than predicted by extrapolations with other ion species (Famá et al. 2008; Johnson et al., 2009, Cassidy et al., 2013). This has some implications for the Jovian system, in particular for the atmosphere of Europa. The enhanced sputtering yields reported here imply that the pressure of sputtered $\mathrm{O}_{2}, \mathrm{H}_{2}$, and $\mathrm{H}_{2} \mathrm{O}$ at Europa's surface exceed previous model predictions by roughly $30 \%$, all else being equal. Comparing the sputtering yield from $\mathrm{SF}^{+}$ to $\mathrm{S}^{+}$and $\mathrm{F}^{+}$between 20 and $70 \mathrm{keV}$, we find that sputtering induced by molecules is two times more efficient than expected from the sputtering yield of monoatomic species. This is the same finding as in previous sputtering experiments by Galli et al. (2017) for atomic versus molecular oxygen.

\section{Acknowledgements}

The work in this paper has been partially performed in the context of the activities of the ISSI International Team Nr. 322, www.issibern. ch/teams/exospherejuice/. We also would like to thank G. Bodmer, J. Gon-

seth, and A. Etter for their relentless support of the scientific work at the MEFISTO facility. 
Baragiola, R.A. Vidal, R.A., Svendsen, W., Schou, J., Shi, M., Bahr, D.A., Atteberry, C.L., 2003. Sputtering of water ice. Nuclear Instruments and Methods in Physics Research B 209, 294-303.

Cassidy, T.A., Johnson R.E., 2005. Monte Carlo model of sputtering and other ejection processes within a regolith. Icarus 176, 499-507.

Cassidy, T., Coll, P., Raulin, F., Carlson, R.W., Johnson, R.E., Loeffler, M.J., Hand, K.P., Baragiola, R.A., 2010. Radiolysis and Photolysis of Icy Satellite Surfaces: Experiments and Theory. Space Science Reviews. doi:10.1007/s11214-009-9625-3.

Cassidy, T.A., Paranicas, C.P., Shirley, J.H., DaltonIII, J.B., Teolis, B.D., Johnson, R.E., Kamp, L., Hendrix, A.R., 2013. Magnetospheric ion sputtering and water ice grain size at Europa. Planetary and Space Science 77, 64-73.

Cooper, J.F., Johnson, R.E., Mauk, B.H., Garrett, H.B., Gehrels, N., 2001. Energetic Ion and Electron Irradiation of the Icy Galilean Satellites. Icarus 149, 133-159.

Famá, M., Shi, J., Baragiola, R.A., 2008. Sputtering of ice by low-energy ions. Surface Science 602, 156.

Farenzena, L.S., Iza, P., Martinez, R., Fernandez-Lima, F.A., Seperuelo Duarte, E., Faraudo, G.S., Ponciano, C.R., da Silveira E.F., C.R., Homem, M.G.P., Naves de Brito, A., Wien, K., 2005. Electronic sputtering analysis of astrophysical ices. Earth, Moon, and Planets 97, 311-329.

Galli, A., Vorburger, A., Pommerol, A., Wurz, P., Jost, B., Poch, O., Brouet, Y., Tulej, M., Thomas, N., 2016, Surface charging of thick porous water ice layers relevant for ion sputtering experiments, Planetary and Space Sciences, 126, 63-71.

Galli, A., Vorburger, A., Wurz, P., Tulej, M., 2017, Sputtering of water ice films: A re-assessment with singly and doubly charged oxygen and argon ions, molecular oxygen, and electrons, Icarus, 291, 36-45.

Hall, D.T., Strobel, D.F., Feldman, P.D., McGrath, M.A., Weaver, H.A., 1995. Detection of an oxygen atmosphere on Jupiter's moon Europa. Nature 373,677 . 
Hansen, C.J., Shemansky, D.E., Hendrix, A.R., 2005. Cassini UVIS observations of Europa's oxygen atmosphere and torus. Icarus 176, 305.

Haring, R.A., Kolfschoten, A.W., De Vries, A.E., 1984. Chemical sputtering by keV ions. Nuclear Instruments and Methods in Physics Research, B2, $544-549$.

Haring, R.A., Pedrys, R., Oostra, D.J., Haring, A., De Vries, A.E., 1984. Reactive sputtering of simple condensed gases by keV ions III: kinetic energy distributions. Nuclear Instruments and Methods in Physics Research, B5, 483-488.

Ip, W.-H., Williams, D.J., McEntire, R.W., Mauk, B.H., 1998. Ion sputtering and surface erosion at Europa. Geophysical Research Letters, 25, 829-832, doi:10.1029/98GL00472.

Johnson, R.E., 1989. Application of Laboratory Data to the Sputtering of a Planetary Regolith. Icarus 78, 206-210.

Johnson, R.E., Carlson, R.W., Cooper, J.F., Paranicas, C., Moore, M.H., Wong, M.C., 2004. Radiation effects on the surfaces of the Galilean satellites. In: Bagenal, F. (Ed.), Jupiter: Atmosphere, Satellites and Magnetosphere. University of Arizona Press, Tucson, USA.

Johnson, R.E., Burger, M.H., Cassidy, T.A., Leblanc, F., Marconi, M., Smyth, W.H., 2009. Composition and Detection of Europas SputterInduced Atmosphere. In: Pappalardo, R.T., McKinnon, W.B., Khurana, K.K. (Eds.), Europa. University of Arizona Press, Tucson.

Marti, A., Schletti, R., Wurz, P., Bochsler, P., 2001. Calibration facility for solar wind plasma instrumentation. Review of Scientific Instruments 72, 1354. doi:10.1063/1.1340020.

Mitchell, E.H., Raut, U., Teolis, B.D., Baragiola, R.A., 2017. Porosity effects on crystallization kinetics of amorphous solid water: Implications for cold icy objects in the outer solar system. Icarus 285, 291-299.

Muntean, E.A., Lacerda, P., Field, T.A., Fitzsimmons, A., Fraser, W.C., Hunniford, A.C., McCullough, R.W., 2016. A laboratory study of water ice erosion by low-energy ions. Monthly Notices of the Royal Astronomical Society 462, 3361-3367. 
Paranicas, C., Carlson, R. W., Johnson, R.E., 2001. Electron bombardment of Europa. Geophysical Research Letters 28, 673-676, doi:10.1029/2000GL012320.

Paranicas, C., Mauk, B.H., Ratliff, J.M., Cohen, C., Johnson, R.E., 2002. The ion environment near Europa and its role in surface energetics. Geophysical Research Letters 29, 18.

Plainaki, C., Milillo, A., Mura, A., Orsini, S., Massetti, S., Cassidy, T., 2012. The role of sputtering and radiolysis in the generation of Europa exosphere. Icarus, 218, 956.

Reimann, C.T., Boring, J.W., Johnson, R.E., Garrett, J.W., Farmer, K.R., 1984. Ion-induced molecular ejection from $\mathrm{D}_{2} \mathrm{O}$ ice. Surface Science 147, $227-240$.

Roth, L., Saur, J., Retherford, K.D., Strobel, D.F., Feldman, P.D., McGrath, M.A., Spencer, J.R., Blcker, A., Ivchenko, N., 2016. Europa's far ultraviolet oxygen aurora from a comprehensive set of HST observations. Journal of Geophysical Research 121, 2143-2170.

Shematovich, V. I., Johnson, R. E., Cooper, J. F., Wong, M. C., 2005. Surface-bounded atmosphere of Europa. Icarus 173, 480.

Shi, M., Baragiola, R. A., Grosjean, D. E., Johnson, R. E., Jurac, S., Schou, J., 1995. Sputtering of water ice surfaces and the production of extended neutral atmospheres. Journal of Geophysical Research 100, 26,387-26,395.

Smyth, W.H. and Marconi, M.L., 2006. Europa's atmosphere, gas tori, and magnetospheric implications. Icarus 181, 510-526.

Sigmund, P. 1969. Theory of sputtering. I. Sputtering yield of amorphous and polycrystalline targets. Physical Review 184, 383-416.

Teolis, B.D., Vidal, R.A., Shi, J., Baragiola, R.A., 2005. Mechanisms of $\mathrm{O}_{2}$ sputtering from water ice by keV ions. Physical Review B 72, 245422.

Teolis, B.D., Shi, J., Baragiola, R.A., 2009. Formation, trapping, and ejection of radiolytic $\mathrm{O}_{2}$ from ion-irradiated water ice studied by sputter depth profiling. The Journal of Chemical Physics 130, 134704. 
Vidal R.A., Teolis, B.D., Baragiola, R.A., 2005. Angular dependence of the sputtering yield of water ice by $100 \mathrm{keV}$ proton bombardment. Surface Science 588, 1-5.

Vorburger, A., Wurz, P., 2018. Europa's Ice-Related Atmosphere: The Sputter Contribution. Icarus 311, 135-145.

Ziegler, J.F., Biersack, J.P., Littmark, U., 1985. The stopping and range of ions in matter. Pergamon, New York.

Ziegler, J.F., Biersack, J.P., Ziegler, M. D., 2008. SRIM - The Stopping and Range of Ions in Matter, Vol. 5. SRIM Co., Chester, MD. 